\title{
THE SOLAR NEIGHBORHOOD. XI. THE TRIGONOMETRIC PARALLAX OF SCR 1845-6357
}

\author{
Niall R. Deacon and Nigel C. Hambly \\ Institute for Astronomy, University of Edinburgh, Blackford Hill, Edinburgh EH9 3HJ, UK \\ AND \\ Todd J. Henry, John P. Subasavage, Misty A. Brown, and Wei-Chun Jao \\ Department of Physics and Astronomy, Georgia State University, Atlanta, GA 30302-4106 \\ Received 2004 August 6; accepted 2004 September 21
}

\begin{abstract}
We present a trigonometric parallax for the nearby star SCR 1845-6357, an extremely red, high proper motion object discovered by Hambly et al. and identified via accurate photoelectric photometry and spectroscopy to be an M8.5 dwarf with a photometric parallax of $4.6 \pm 0.8 \mathrm{pc}$ by Henry et al. Using methods similar to those described in Deacon \& Hambly, we have derived a full astrometric solution from SuperCOSMOS scans of eight survey and nonsurvey Schmidt photographs held in the United Kingdom Schmidt Telescope Unit plate library. We calculate the trigonometric parallax to be $\pi=282 \pm 23$ mas, yielding a distance of $3.5 \pm 0.3 \mathrm{pc}$, which implies an absolute $K_{S}$ magnitude of 10.79. This distance calculation places SCR $1845-6357$ as the 16th closest stellar system to the Sun.
\end{abstract}

Key words: solar neighborhood — stars: distances — stars: late-type — stars: low-mass, brown dwarfs

\section{INTRODUCTION}

There has been a recent flurry of discoveries of stars within the local solar neighborhood. Some have been discovered as companions to well-known nearby stars (e.g., $\epsilon$ Indi B/C; see McCaughrean et al. 2004 and references therein), whereas others are entirely new systems (e.g., Teegarden et al. 2003). The Research Consortium on Nearby Stars (RECONS) group seeks to identify and study all systems within $10 \mathrm{pc}$ of the Sun. Previous papers of this series have used a variety of photometric and spectroscopic techniques to identify and study samples of nearby stars and describe discovery of companions to known nearby stars and identification of entirely new systems. Most recently, a new initiative (Hambly et al. 2004) identified five nearby, high proper motion stars, two of which have initial photometric parallaxes within the RECONS horizon. The nearest of these, SCR 1845-6357 (hereafter the target) was shown subsequently to have a photometric parallax of $4.6 \pm 0.8 \mathrm{pc}$ (Henry et al. 2004). Photometric data for the target are reproduced in Table 1. These show that the object is very red; Henry et al. (2004) classified the target as an M8.5 dwarf.

\section{OBSERVATIONAL DATA AND REDUCTION}

Luckily there is a wealth of astrometric data available for this object, partly because it lies in the overlap region between the standard Schmidt photographic survey fields and partly because nonsurvey programs have fortuitously observed the field containing the target frequently and over a long time baseline. The target was identified on two United Kingdom Schmidt Telescope (UKST) $R$ survey plates and one European Southern Observatory (ESO) survey $R$ plate. These had already been scanned as part of the SuperCOSMOS Sky Survey (Hambly et al. 2001b and references therein). In addition, six UKST nonsurvey $R$ photographs from the UKST Plate Library were selected for their image quality and useful parallax factors and were scanned especially for this study using SuperCOSMOS. Hence, a total of nine Schmidt photographs were used; details of these are shown in Table 2. These also provide a 9 yr baseline, ideal for measuring an accurate proper motion.

Each plate was scanned individually on SuperCOSMOS and processed using standard methods (Hambly et al. 2001b and references therein). Global astrometric plate solutions result in systematic errors on the order of $\sim 0{ }^{\prime \prime} 2$ in absolute positions. Hence, it is necessary to correct for these; a local linear plate model with respect to the array of mean positions from all measures was employed for this purpose. A sample of circular, single, stellar images that are not affected by proximity to bright stars were selected as local astrometric reference stars. These reference stars were used to fit linear models for each of the plates with respect to the array of mean reference star positions. The residual errors from the reference stars after these models are applied give an indication of the astrometric quality of each plate; as a result of this test, one of the nonsurvey plates was excluded from the rest of the study because of poor astrometric quality (see Table 2).

It is also important that the maximum distance of reference stars from the target is carefully chosen. Too small a distance and the number of reference stars will be too few; too large and nonlinear plate effects may leave systematic errors in the linear fit (indicated by an increase in the rms error). In order to choose the maximum reference star distance correctly, several different selections were made, and the average rms errors (the mean of all the errors from all the plates) from the local linear plate fit were noted along with the number of reference stars. The results are shown in Table 3. We chose the maximum extent available $\left(20^{\prime}\right)$ from the area in common between both plates, since there is no indication of an increase in systematic errors up to this value.

\section{RESULTS}

Once the process described in $\S 2$ was carried out, plate-toplate systematic errors were eliminated, the relative astrometric quality of each plate with respect to the mean positional measures was determined, and it was possible to apply a full 
TABLE 1

Рнотоmetric Data on SCR $1845-6357$

\begin{tabular}{ccccccccc}
\hline \hline$B_{\mathrm{J}}$ & $R_{59}$ & $I_{\mathrm{N}}$ & $J$ & $H$ & $K_{s}$ & $V$ & $R_{\mathrm{C}}$ & $I_{\mathrm{C}}$ \\
\hline 19.05 & 16.33 & 12.53 & 9.54 & 8.97 & 8.51 & 17.40 & 15.00 & 12.47 \\
\hline
\end{tabular}

NoтE.-The $J H K$ magnitudes are taken from the Two Micron All Sky Survey; $V, R$, and $I$ measurements are from Henry et al. 2004. Note that the $B_{\mathrm{J}}$ measurement comes from a plate on which the target image is deblended and hence may not be as accurate as the other measurements.

TABLE 2

Schmidt Photographs Used in this Study

\begin{tabular}{|c|c|c|c|c|c|c|c|c|c|}
\hline Plate Number & Date & LST & $\begin{array}{l}\text { Zenith Angle } \\
\text { (deg) }\end{array}$ & Emulsion & Filter & $\begin{array}{l}\text { Exposure } \\
\text { (minutes) }\end{array}$ & $\begin{array}{c}\sigma_{x} \\
\text { (mas) }\end{array}$ & $\begin{array}{c}\sigma_{y} \\
\text { (mas) }\end{array}$ & Material \\
\hline \multicolumn{10}{|c|}{ Plates Used } \\
\hline ESOR6887 .......... & 1987 Apr 30 & 1713 & 37.7 & IIIaF & RG630 & 120 & 46 & 64 & Glass copy of ESO survey plate \\
\hline OR13751 .............. & 1990 Jun 20 & 1843 & 32.7 & IIIaF & OG590 & 60 & 41 & 42 & Original nonsurvey plate \\
\hline OR14370 .............. & 1991 Jun 16 & 1831 & 32.8 & $\mathrm{IIIaF}$ & OG590 & 55 & 40 & 39 & Original nonsurvey plate \\
\hline OR15689 .............. & 1993 Aug 12 & 1746 & 34.0 & IIIaF & OG590 & 55 & 40 & 42 & Original survey plate \\
\hline OR16753 ................ & 1995 Aug 28 & 1850 & 32.7 & IIIaF & OG590 & 80 & 46 & 44 & Original survey plate \\
\hline OR17012 ............... & 1996 Mar 24 & 1648 & 37.5 & 4415 & OG590 & 15 & 48 & 49 & Original nonsurvey film \\
\hline OR17038 .............. & 1996 Apr 15 & 1757 & 33.6 & 4415 & OG590 & 15 & 53 & 56 & Original nonsurvey film \\
\hline OR17256 ............... & 1996 Sep 15 & 1838 & 32.7 & 4415 & OG590 & 15 & 55 & 64 & Original nonsurvey film \\
\hline \multicolumn{10}{|c|}{ Plate Not Used } \\
\hline 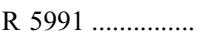 & 1980 May 16 & 2032 & 36.7 & $\mathrm{IIIaF}$ & RG630 & 150 & 107 & 92 & Original nonsurvey plate \\
\hline
\end{tabular}

Note.-The relative astrometric quality is indicated by the $\sigma_{x}$ and $\sigma_{y}$ values (see text). One plate was excluded because of poor astrometric quality.

TABLE 3

Selecting the Optimum Maximum Distance of Reference Stars from the Target

\begin{tabular}{|c|c|c|}
\hline $\begin{array}{l}\text { Maximum Distance from Target } \\
\text { (arcmin) }\end{array}$ & $\begin{array}{l}\text { Average rms Error } \\
\text { (mas) }\end{array}$ & Number of Reference Stars \\
\hline $20 \ldots \ldots \ldots$ & 56 & 108 \\
\hline 18 & 56 & 108 \\
\hline 16 & 56 & 98 \\
\hline 14 & 58 & 77 \\
\hline $12 \ldots \ldots \ldots$ & 59 & 56 \\
\hline 10 & 58 & 41 \\
\hline
\end{tabular}

Note.-The number of reference stars does not increase as the square of the maximum radius because of plate boundary cutoffs.

weighted astrometric fit to the target and reference stars. Equations (1) and (2) show the astrometric models for right ascension and declination, respectively (where $f_{\alpha}$ and $f_{\delta}$ are the parallax factors in right ascension and declination):

$$
\begin{gathered}
\alpha=\alpha_{o}+\mu_{\alpha} t+\pi f_{\alpha}, \\
\delta=\delta_{o}+\mu_{\delta} t+\pi f_{\delta} .
\end{gathered}
$$

The error-weighted design matrix formed from these equations was solved via singular value decomposition to yield a least-squares fit; we employed numerical routines from the SLALIB positional astronomy library (Wallace 2003). The results for the target (along with $\chi_{\nu}^{2}$, i.e., $\chi^{2}$ normalized per degree of freedom) are shown in Table 4 . The total proper motion is 2 ". $64 \pm 0 . \prime 0082 \mathrm{yr}^{-1}$ with a position angle of $74^{\circ} .9$; these compare with the values of $2^{\prime \prime} .56 \pm 0.013 \mathrm{yr}^{-1}$ and 74.8 quoted in Hambly et al. (2004). The proper-motion measurements differ, but this may be due to systematic errors in either measurement or because Hambly et al. (2004) did not include a parallax in their astrometric solution. The trigonometric parallax was found to be $\pi=282 \pm 23$ mas. Figure 1 shows the deviation in position of the target from its proper motion. Figure 2 shows the parallax ellipse traced out by the target star.

As a test of the astrometry, each reference star was also run through the same astrometric model-fitting procedure, and the proper motions and parallaxes of the reference stars were found. These are plotted in Figure 3, along with the target. It is clear that the target is well separated from the mass of reference stars. 
TABLE 4

Full Astrometric Solution for SCR 1845-6357

\begin{tabular}{|c|c|}
\hline Parameter & Fitted Value \\
\hline R.A. on 2000 Jan 1 & $1845050.2008 \pm 0.0062$ \\
\hline Decl. on 2000 Jan 1 & $-635747.355 \pm 0.0435$ \\
\hline$\mu_{\alpha} \cos \delta$ & 2 ".5495 $\pm 0.0055 \mathrm{yr}^{-1}$ \\
\hline$\mu_{\delta}$ & $0.6874 \pm 0.0061 \mathrm{yr}^{-1}$ \\
\hline$\pi$ & $282 \pm 23$ mas \\
\hline$\chi_{\nu}^{2}$ & 0.49 \\
\hline
\end{tabular}

Note.- Units of right ascension are hours, minutes, and seconds, and units of declination are degrees, arcminutes, and arcseconds. Note the $\chi_{\nu}^{2}$ indicating a good fit to the model.

In general the reference stars have proper motions and parallaxes consistent with zero (and a mean value of $\chi_{\nu}^{2} \sim 0.72$, indicating good model fits) and hence are good reference stars. To further investigate the reference star distances and for the purposes of correcting the measured (i.e., relative) parallax to an absolute parallax, the mean $B_{\mathrm{J}}-R_{59}$ and $R_{59}$ for the reference stars were found. The mean $B_{\mathrm{J}}-R_{59}$ calculation was then used to find that the mean spectral type (assuming they are dwarfs) for the reference stars is G5 (Zombeck 1990). From this the mean expected absolute $R_{59}$ magnitude (Zombeck 1990) can be deduced, which, combined with the mean $R_{59}$ magnitude, yields the mean expected distance of the reference stars.
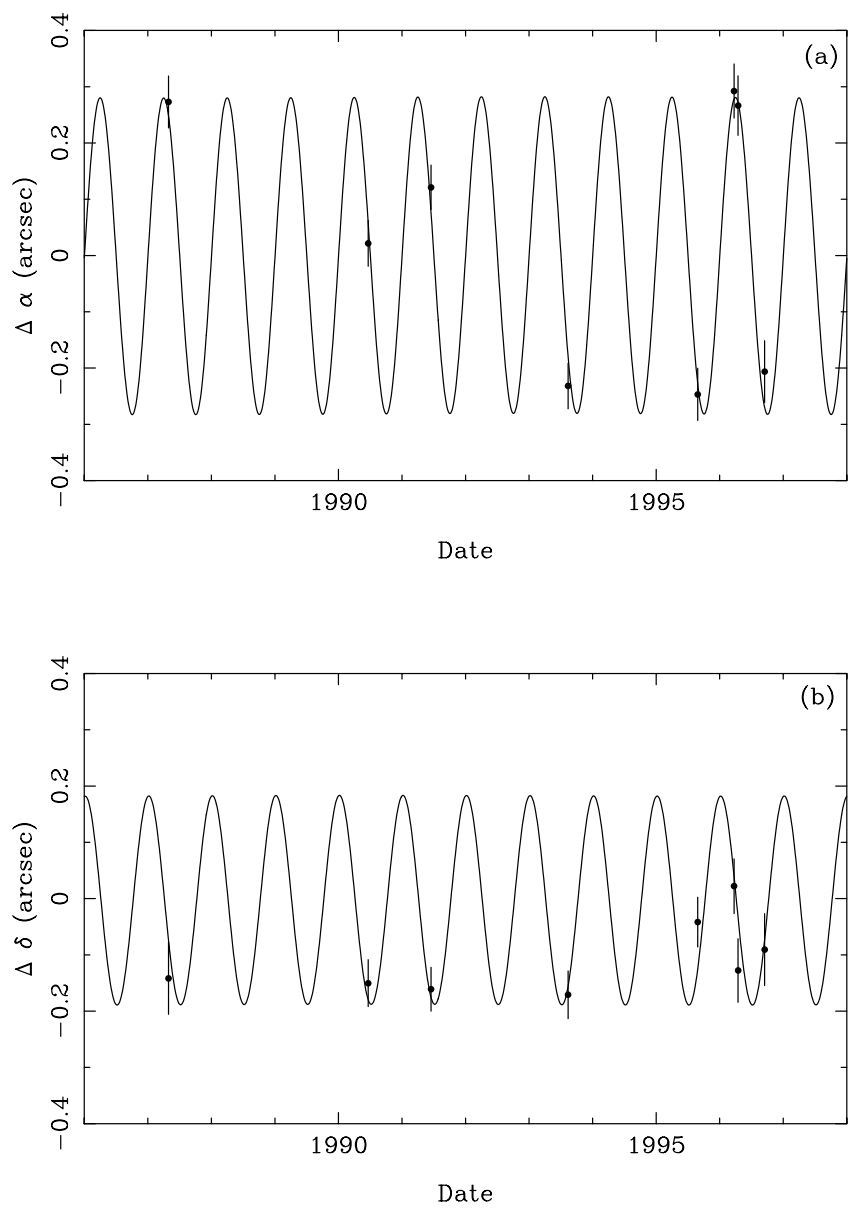

FIG. 1.-Deviation from the proper motion in (a) right ascension and $(b)$ declination vs. time. The line shown is the path of parallax motion predicted by the astrometric solution.

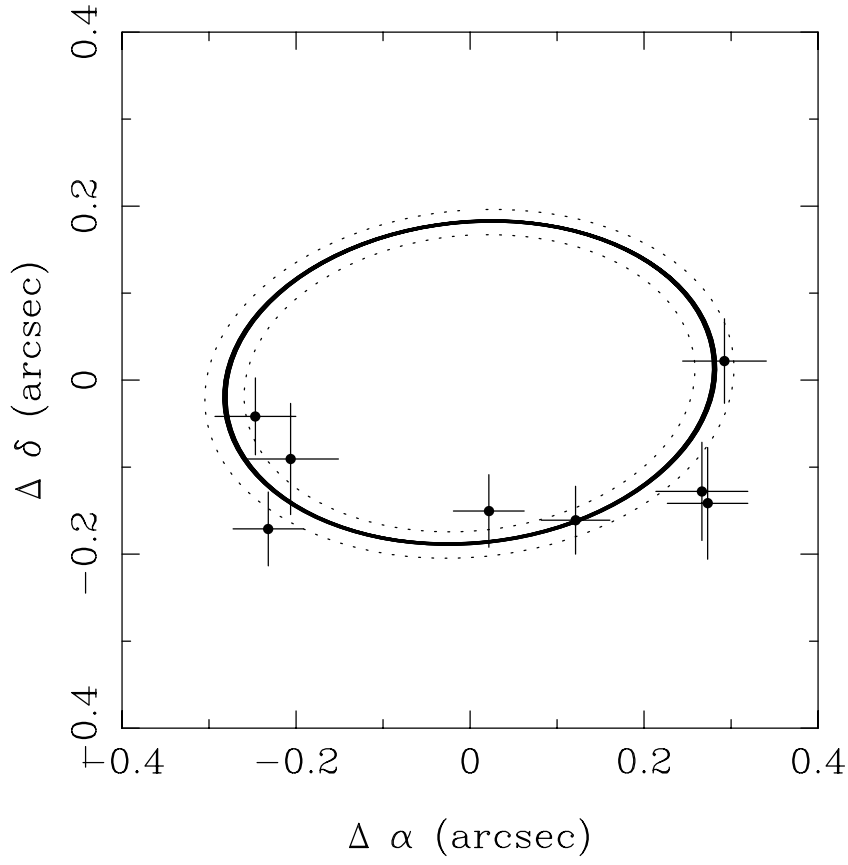

Fig. 2.-Parallax ellipse traced out by the target. The dotted lines represent $1 \sigma$ upper and lower limits on the parallax.

The mean expected trigonometric parallax of the reference stars was thus found to be $\pi=0.7$ mas. This is clearly insignificant compared to the formal error on our parallax for the target, so we made no correction from relative to absolute parallax.

To further test that the method used was sound, 100 sets of simulated data were created. Each had the same astrometric parameters as the target, and each data point was given a random Gaussian error calculated from the rms error estimates of the particular plate. These were then run through the astrometric solution-fitting program. No significant offsets were found, and the error on the parallax calculated from the scatter of the simulated data solutions was in good agreement with that predicted by the astrometric solution.

Finally, we examined differential color refraction (DCR) effects between the reference stars and the target. In Figure 4 we show model predictions for the coefficient of refraction, $R$, for stars of various synthetic photographic color. We used methods described in Hambly et al. (2001a) and references

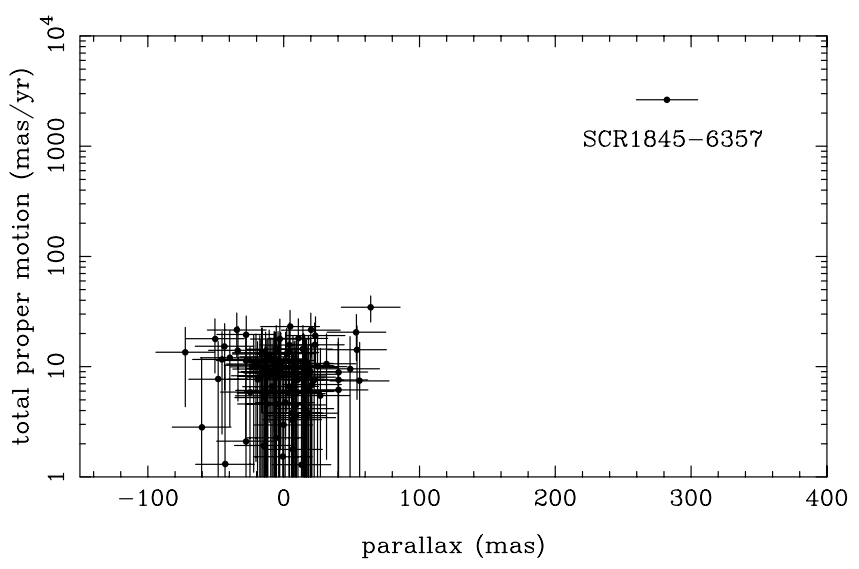

FIG. 3.-Comparing the fitted parallax and proper motions for the reference stars with SCR $1845-6357$. 


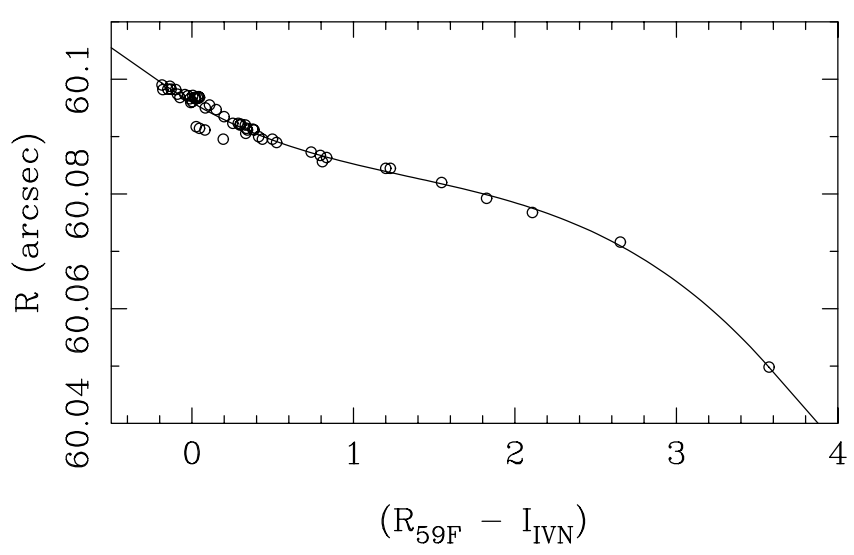

FIG. 4. - Computational models (after Hambly et al. 2001a and references therein) for the coefficient of refraction $R$ at an air mass of 1.5 as a function of synthetic photographic $R-I$ color. Data are from a spectrophotometric atlas, except for the point at $(R-I, R)=(3.6,60.05)$, for which we have used the spectrum for SCR $1845-6357$ presented in Henry et al. (2004). The solid line is a polynomial fit to the data points.

therein; we additionally used the flux-calibrated spectrum of SCR 1845-6357 presented in Henry et al. (2004) to compute $R_{59}$ and synthetic $R_{59}-I_{\mathrm{IVN}}$ for the extremely red target. The reference stars are taken as an ensemble of points that typically have $R_{59}-I_{\mathrm{IVN}} \sim 0.5$, whereas the target has $R_{59}-I_{\mathrm{IVN}}=3.6$ computed from the spectrum and 3.75 from the photographic photometry listed in Table 1. (These colors are consistent within the photographic photometric errors of $\sim 0.1 \mathrm{mag}$ for $R_{59}-I_{\mathrm{IVN}}$.) These colors indicate a difference in the refraction coefficient of $\Delta R \sim 35$ mas at an air mass of 1.5 (Fig. 4). This difference changes negligibly with air mass; i.e., recomputations at 1.0 and 2.0 air masses show a zero-point shift in the locus of Figure 4 but no significant change in the $\Delta R$ value for the two colors. However, the quantity of most relevance is, of course, how this differential refraction coefficient translates into image displacement at the different hour angles and zenith distances of the observations in Table 2. Typically, the DCR shift for right ascension is \pm 5 mas and for declination is \pm 1.5 mas; the largest change between any two observations is 10 mas for right ascension and 3 mas for declination because all of the plates used are within $2 \mathrm{hr}$ of the meridian, so DCR is minimal. Hence, we feel justified in neglecting these corrections in our astro- metric model, since the positional uncertainties dominate systematic effects due to DCR.

\section{DISCUSSION}

The calculated trigonometric parallax for the target gives a distance of $3.5 \pm 0.3 \mathrm{pc}$. Consulting the RECONS list of nearby stars, ${ }^{1}$ we find that this makes SCR $1845-6357$ the 16 th nearest stellar system to the Sun. The upper and lower $1 \sigma$ error boundaries would make it the 23rd and 10th nearest stellar system, respectively. We note that the RECONS photometric parallax is $4.6 \pm 0.3 \mathrm{pc}$ (Henry et al. 2004); hence, our new trigonometric parallax puts this object $2.8 \sigma$ nearer than was first estimated. Hambly et al. (2004) estimated the distance to be $3.5 \pm 0.7 \mathrm{pc}$; we expect the true distance of the target to be between the estimate given here and that of Henry et al. (2004).

\section{CONCLUSION}

We have measured the trigonometric parallax of SCR $1845-6357$ and found it to be the 16th nearest stellar system to the Sun. This demonstrates that the wealth of astrometric information on the many plates taken over the past 50 years can yield new insight into the nearby star population and, in particular, permit the determination of valuable trigonometric parallaxes.

The authors would like to thank Sue Tritton and Mike Read for their help in selecting plates and Harvey MacGillivray and Eve Thomson for their prompt scanning of the material on SuperCOSMOS. SuperCOSMOS is funded by a grant from the UK Particle Physics and Astronomy Research Council. This publication makes use of data products from the Two Micron All Sky Survey, which is a joint project of the University of Massachusetts and the Infrared Processing and Analysis Center/ California Institute of Technology, funded by the National Aeronautics and Space Administration and the National Science Foundation.

${ }^{1}$ See http://www.chara.gsu.edu/RECONS.

\section{REFERENCES}

Hambly, N. C., Davenhall, A. C., Irwin, M. J., \& MacGillivray, H. T. 2001a, MNRAS, 326, 1315

Hambly, N. C., et al. 2001b, MNRAS, 326, 1279

Hambly, N. C., Henry, T. J., Subasavage, J. P., Brown, M. A., \& Jao, W.-C. 2004, AJ, 128, 437

Henry, T. J., Subasavage, J. P., Brown, M. A., Beaulieu, T. D., Jao, W.-C., \& Hambly, N. C. 2004, AJ, 128, 2460

McCaughrean, M. J., Close, L. M., Scholz, R.-D., Lenzen, R., Biller, B., Brandner, W., Hartung, M., \& Lodieu, N. 2004, A\&A, 413, 1029
Teegarden, B. J., et al. 2003, ApJ, 589, L51

Wallace, P. T. 2003, SLALIB - Postitional Astronomy Library 2.4-12 Programmer's Manual (Starlink User Note 67.61) (Chilton: CCLRC/Rutherford Appleton Laboratory, PPARC)

Zombeck, M. V. 1990, Handbook of Space Astronomy and Astrophysics (2nd ed; Cambridge: Cambridge Univ. Press) 